\title{
The Speed of Light Is Slowed Down as It Travels Near the Surface of an Obstacle
}

\author{
Farhad Vedad
}

\section{ABSTRACT}

The speed of propagation of light that passes through a medium at a slower speed determines the refractive index with respect to the wavelength. Subsequently, the refractive index of vacuum (free space) that light travels at speed $\mathrm{c}$ is 1 by definition.

This study proposes that the speed of light is slowed down in an inhomogeneous space close to the surface of the obstacles, including the edges of the apertures. Furthermore, the inhomogeneous refractive index near these surfaces will increase as the distance from the object is reduced. Therefore, light propagates through apertures as it encounters a medium with a variable refractive index.

Keywords: Speed of Light, Diffraction, Refraction, Inhomogeneous Space, Slow Light, Inhomogeneous Refractive Index.

Published Online: July 13, 2021

ISSN: $2684-4451$

DOI : $10.24018 /$ ejphysics.2021.3.4.90

\section{Farhad Vedad*}

Graduate Faculty of Nuclear Engineering and Physics, Amirkabir University of Technology (Tehran Polytechnic), Iran. (e-mail: farhad.vedad ${ }^{@}$ gmail.com)

*Corresponding Author

\section{INTRODUCTION}

Concerning the propagation of light, there are two concepts of phase and group velocity. The phase velocity of light in a medium has an inverse relationship with the refractive index $n$.

$v_{p}=\frac{c}{n}$

where $c$ is the speed of light in the vacuum. However, in anomalous dispersion, the group velocity is not equal to the phase velocity and it may exceed c. [1].

Furthermore, one of the simplified models describing the relationship for the refractive index versus the wavelength of light is Cauchy dispersion equation that can be used for various glasses and also a nematic liquid crystal such as cyano-biphenyl in the visible region of the spectrum ([2] and [3]) (See Fig. 1).

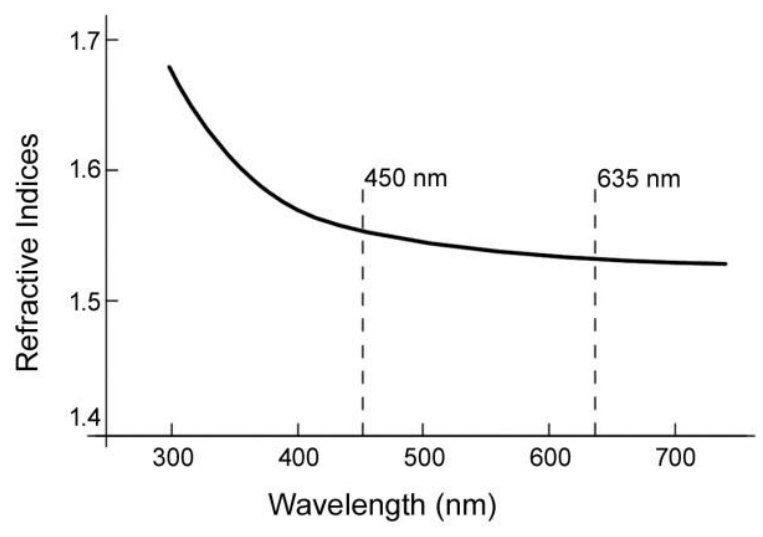

Fig. 1. Cauchy model.
On the other hand, the concept of the fractal inhomogeneous space near the surfaces of the obstacles suggests that the space close to the edges of the apertures is inhomogeneous and maintaining an exponential up-chirp function in accordance with a variable refractive index. Therefore the refractive index near the surface of an object will be higher, and will reduce as the distance from the object is increased [4].

If this concept is correct, then the light should be slowed down when it travels through an aperture as it encounters a medium.

\section{EXPERIMENTAL METHOD}

It is presumable that the eventual time delay of light traveling through an opaque aperture in the diffraction experiment is too insignificant that it is not denoted in any measurement or estimation model. Consequently, using high-precision test instruments will not improve the signal processing to detect this imperceptible time delay from the low dimensional noise either.

Despite of this, a series of lined up apertures may increase the value of the time delay of the light signal. Eventually, this complex of apertures will act equal to a medium with refractive index $n>1$, and therefore, it can be considered as a unit delay block (UDB).

Fig. 2 shows the setup of the refractometer for measuring the time delay caused by a UDB.

Measurements were performed using nanosecond pulsed lasers at $450 \mathrm{~nm}, 520 \mathrm{~nm}$, and $640 \mathrm{~nm}$ triggered externally rep. rate $20 \mathrm{MHz}, 20 \mathrm{R} / 80 \mathrm{~T}$ non-polarizing beam splitter, a couple of $\mathrm{Si}$ biased detectors, and a $2 \mathrm{CH}$ Oscilloscope $250 \mathrm{MHz}$. 


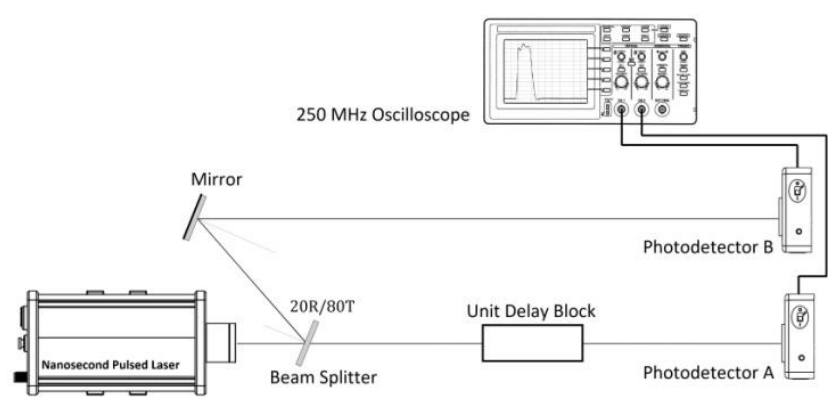

Fig. 2. Schematic diagram of the refractometer by using a unit delay block.

In the first step, I use a $\mathrm{K} 9$ crystal cube (cross-section of $20 \times 60 \mathrm{~mm}$, and length of $80 \mathrm{~mm}$ ) with the refractive index of $1.5248(450 \mathrm{~nm}), 1.5197(520 \mathrm{~nm})$, and $1.5144(640 \mathrm{~nm})$ as a UDB to double-check the experimental set-up and prevent any error.

Then, I repeated an equivalent experiment by using a series of apertures as a UDB to estimate the time delay of the light signals.

\section{Two HundRED Lined Up Single SLITS WiTH METAL BARRIERS}

To measure the time delay caused by an opaque aperture, I lined up two hundred single slits with a width of $0.15 \mathrm{~mm}$ at a length of $450 \mathrm{~mm}$ and later I repeated the equivalent experiment with a width of $0.20 \mathrm{~mm}$.

To produce that complex of the lined up single slits, I used 400 steel die blades in two rows with a gap in between to produce a unit delay block with 200 single slits at a length of $450 \mathrm{~mm}$ (UDB-200/450). Table I demonstrates the time delay caused by two hundred single slits, as well as a single slit.

TABLE I: TIME DELAY CAUSED BY UDB-200/450 IN COMPARISON WITH A SINGLE APERTURE

\begin{tabular}{ccccc}
\hline \multirow{2}{*}{$\begin{array}{c}\text { Wavelength } \\
(\mathrm{nm})\end{array}$} & \multicolumn{2}{c}{$\begin{array}{c}\text { Time delay by 200 single } \\
\text { slits }(\mathrm{ps})\end{array}$} & \multicolumn{2}{c}{$\begin{array}{c}\text { Time delay by every } \\
\text { single slit (ps) }\end{array}$} \\
\cline { 2 - 5 } & Width of & Width of & Width of & Width of \\
& $0.15 \mathrm{~mm}$ & $0.20 \mathrm{~mm}$ & $0.15 \mathrm{~mm}$ & $0.20 \mathrm{~mm}$ \\
\hline 635 & 28.54 & 23.29 & 0.1427 & 0.1164 \\
520 & 34.0 & 26.44 & 0.17 & 0.1322 \\
450 & 40.35 & 30.97 & 0.2017 & 0.1548 \\
\hline
\end{tabular}

Furthermore, Fig. 3 shows the graph of the time delay versus wavelength. Notably, this increase is more pronounced when using a shorter wavelength light source.

As depicted in Fig. 3, the experimental graph satisfies the Cauchy model as well (See Fig. 1).

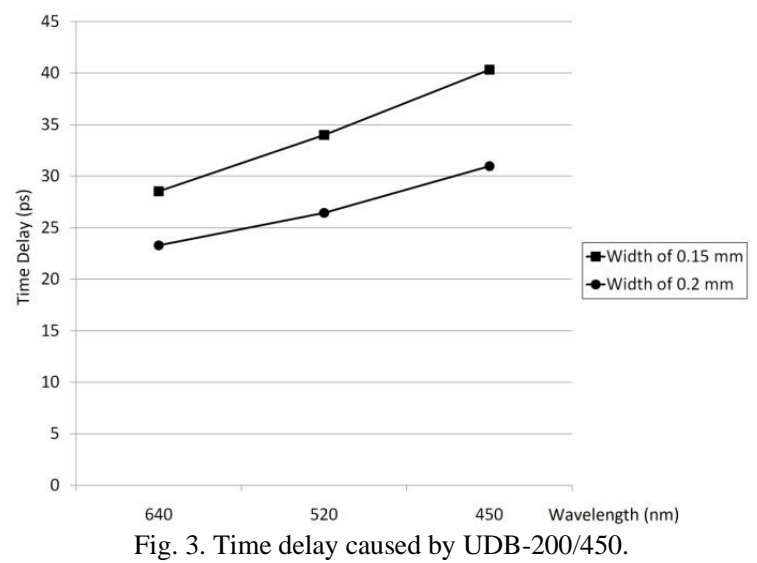

Although Fig. 3 shows the relationship for the time delay versus the wavelength of light, however, it may be implemented by other properties including the multiple reflections and also multiple diffractions in the near-field.

To consider the main parameter underlying time delay of the light signals, I produced two other UDBs with 200 lined up single slits.

\section{Two Hundred Lined Up SingLe SLITS IN A SHORTER LENGTH WITH METAL BARRIERS}

In the experiment of diffraction, the near-field Fresnel approximation at a distance $\mathrm{z}$ in the $(\mathrm{x}, \mathrm{y})$ plane normal to the rectangular coordinates lying in the $\left(\mathrm{x}_{0}, \mathrm{y}_{0}\right)$ plane is expressed by [5]:

$U(x, y, z)$
$=\frac{e^{i k z}}{i \lambda z} e^{i \frac{k}{2 z}\left(x^{2}+y^{2}\right)} \iint_{-\infty}^{\infty} U\left(x_{0}, y_{0}, 0\right) e^{i \frac{k}{2 z}\left(x_{0}^{2}+y_{0}^{2}\right)} e^{-i \frac{k}{z}\left(x_{0} x+y_{0} y\right)} d x_{0} d y_{0}$

Subsequently, diffraction of light may cause different optical path lengths with respect to the wavelength. Furthermore, this increase is more pronounced when increasing the number of the lined up apertures and may cause a time delay in the experiment with UDB-200/450.

To prove that the multiple diffractions didn't underlay the time delay or this effect is not significant enough to produce the results shown in Table 1, the setup was modified with another complex of two hundred single slits at a shorter length of $280 \mathrm{~mm}$ (UDB-200/280) by using 400 razor blades (See also Fig. 4).

The distance between the apertures of UDB-200/450 is $2.25 \mathrm{~mm}$ and it is $1.4 \mathrm{~mm}$ for UDB-200/280. These two distances will influence the appearance of the diffraction pattern in the near-field differently.

Furthermore, the smaller distance between the apertures reduces the amount of the blocked light. Thus, the UDB$200 / 280$ obtains higher outcoming light intensity. It is interesting to note that the experimental result shows that UDB-200/280 and UDB-200/450 caused equal time delay.

\section{Two Hundred Lined Up Single Slits With PlastiC BARRIERS}

The other assumption for implementing the time delay by the complex of two hundred lined up single slits is the multiple reflections. Therefore, the third UDB with the single slits is designed with black plastic razors at the length of $260 \mathrm{~mm}$ (UDB-200/Plastic).

The reflections on smooth facets of the steel razors used for UDB-200/280 and UDB-200/450 are grossly and offspecular. However, the reflections on the rough surfaces of the plastic razors are a combination of both diffuse and offspecular. Notably, in contrast with specular and offspecular reflections, the diffuse reflection is not polarized. [6].

Furthermore, the plastic razors are not as sharp as those steel razors either. Thus, the multiple reflections produced 
by UDB-200/Plastic are totally different than UDB-200/280 and UDB-200/450. Fig. 4 shows the different sections of the steel and plastic razors. (a)

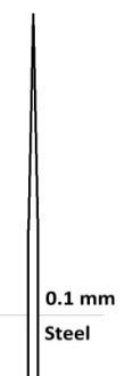

(b)

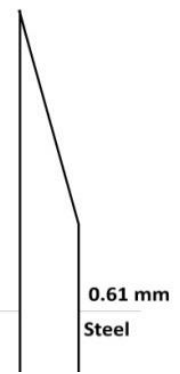

(c)

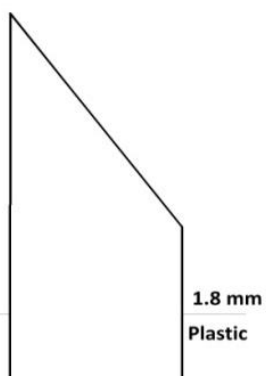

Fig. 4. Sections of the blades. (a) Steel razors used for UDB-200/280, (b) Steel die blades used for UDB-200/450, (c) Black plastic razors used for UDB-200/Plastic.

Finally, the equivalent experiments with three UDBs with steel and plastic barriers defined an equal time delay. The results presented herein demonstrate clearly that the width of the apertures is a dominant factor in determining the time delay.

For the ultimate test, I compared the time delays caused by two UDBs as shown by Fig. 5 .

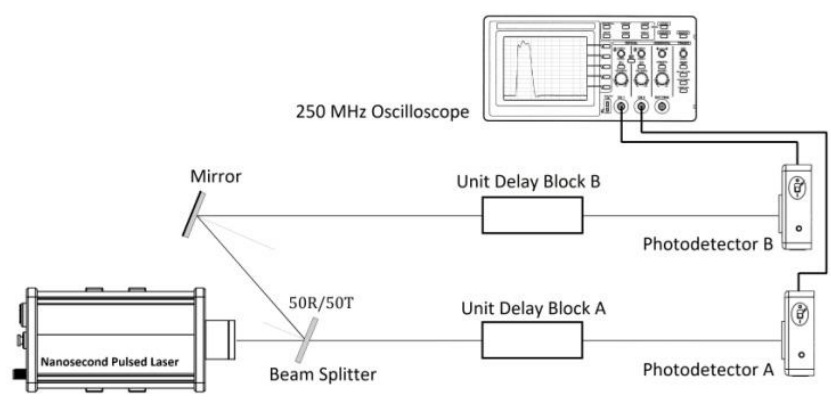

Fig. 5. Schematic diagram of the refractometer by using two unit delay blocks.

In this experiment, a 50R/50T non-polarizing beam splitter is employed to provide an equal intensity of light for both the UDB A and B. Notably, the experimental results demonstrated an equal time delay for both paths A and B regardless of replacing a UDB with another one.

Fig. 6 shows the experiment lab table for estimating the time delays caused by two UDBs. For the experiments performed in this study, UDB-200/450, UDB-200/280, and UDB-200/Plastic were employed (See also Fig. 5).

The experiments with two UDBs demonstrate that the multiple reflections and multiple diffractions are not effective on the time delay caused by the apertures or they considered ignorable. However, there is still a question about the influence of the light intensity detected by the photodetector A as shown by Fig. 2.

The photodetectors $\mathrm{A}$ and $\mathrm{B}$ in these experiments are $\mathrm{Si}$ biased detectors designed for the detection of light signals ranging from $200 \mathrm{~nm}$ to $1100 \mathrm{~nm}$. The responsivity of those photodetectors is $0.15 \mathrm{~A} / \mathrm{W}$ for $450 \mathrm{~nm}, 0.29 \mathrm{~A} / \mathrm{W}$ for 520 $\mathrm{nm}$, and $0.39 \mathrm{~A} / \mathrm{W}$ for $640 \mathrm{~nm}$. The responsivity of a photodiode $(\mathcal{R})$ is defined as a ratio of generated photocurrent I (mA) to the signal optical power $\mathrm{P}(\mathrm{mW})$ at a given wavelength [7]:

$$
\mathcal{R}=\frac{I}{P}
$$

Therefore, the responsivity of a photodiode can be considered as a parameter for underlying the time delay due to the rise time of the photodiode with respect to the wavelength.

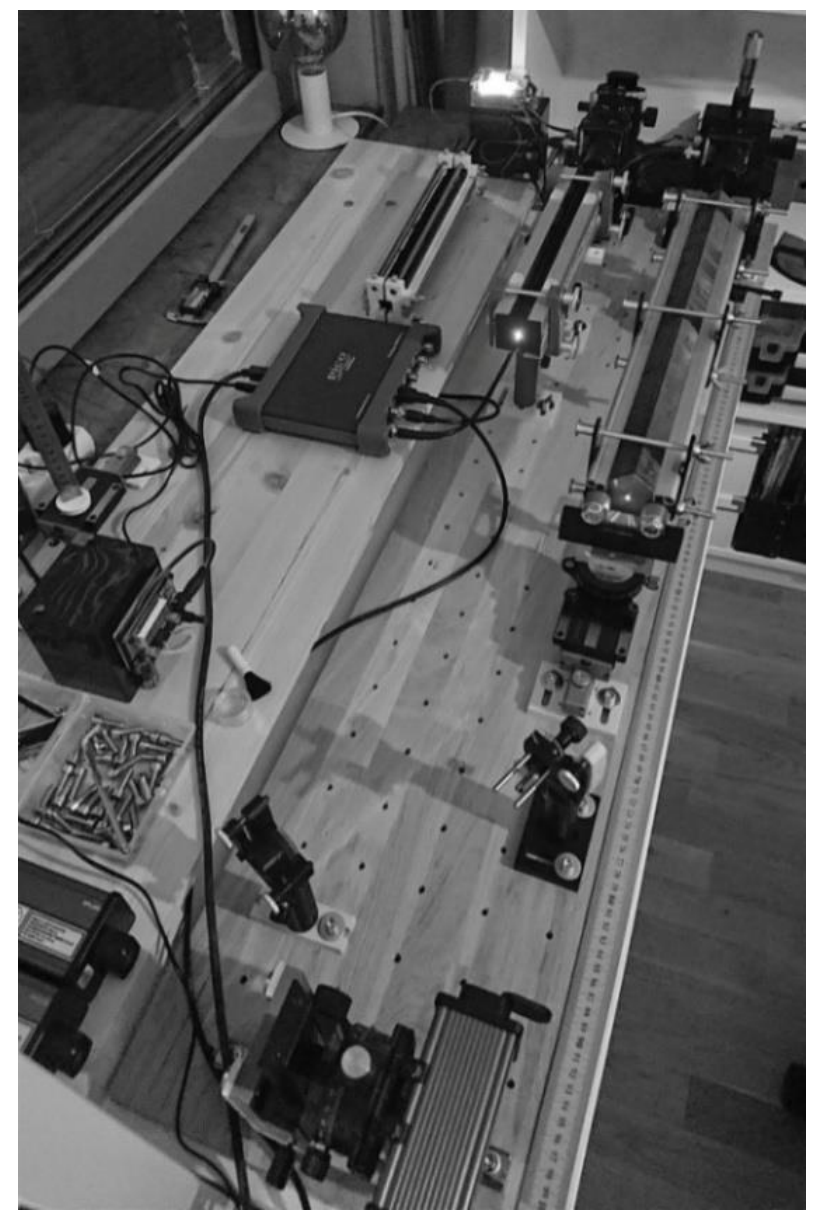

Fig. 6. The experiment lab table for estimation of the time delays.

\section{NARROW LONG TUBES}

To prove that the time delay caused by an opaque aperture in the experiments discussed in the previous sections is not replicating the behavior expected for the responsivity of the photodetector, the setup was modified with narrow long tubes (NLTs).

NLT comprises two aluminum square bar in mat black separated by a narrow gap with a width of $0.15 \mathrm{~mm}$ and also $0.20 \mathrm{~mm}$ at the length of $160 \mathrm{~mm}, 260 \mathrm{~mm}, 360 \mathrm{~mm}, 460$ $\mathrm{mm}$, and $560 \mathrm{~mm}$.

In the experiments with NLTs, the detected light intensity by the photodetector A was more than double comparing with those series of single slits. Notably, the time delay caused by the $260 \mathrm{~mm}$ NLT was nearly equal to those two hundred lined up single slits.

This result predicts that in comparison with UDB200/280, a $280 \mathrm{~mm}$ NLT causes nearly a couple of picoseconds longer time delay, although UDB-200/280 blocks more light. Therefore, the responsivity of the photodetectors is not considered an effective factor in the previous experiments either. 
Fig. 7 shows the time delays caused by a various NLTs with a width of $0.15 \mathrm{~mm}$ versus wavelength.

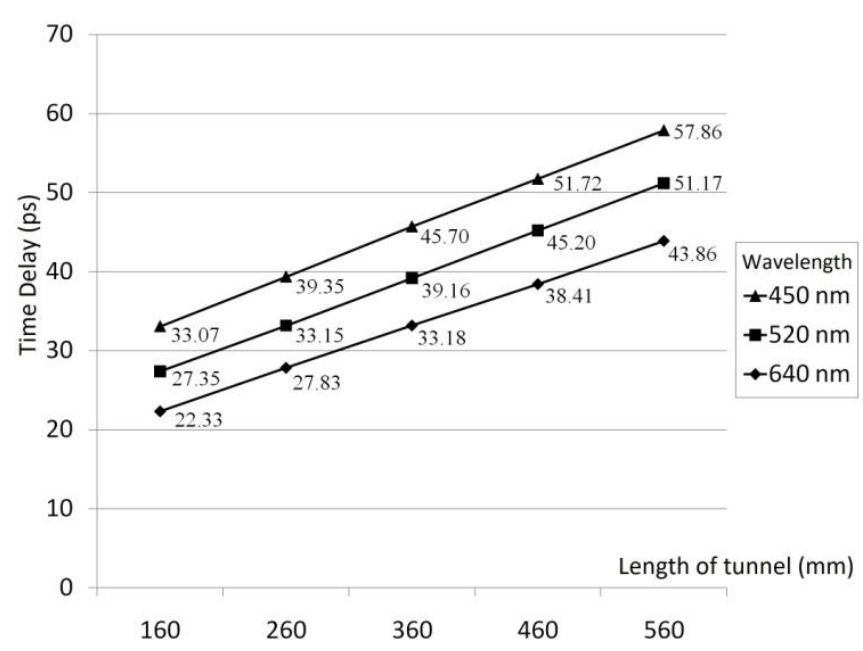

Fig. 7. Time delay caused by NLT with a width of $0.15 \mathrm{~mm}$.

Fig. 8 shows the time delays caused by a various NLTs with a width of $0.20 \mathrm{~mm}$ versus wavelength.

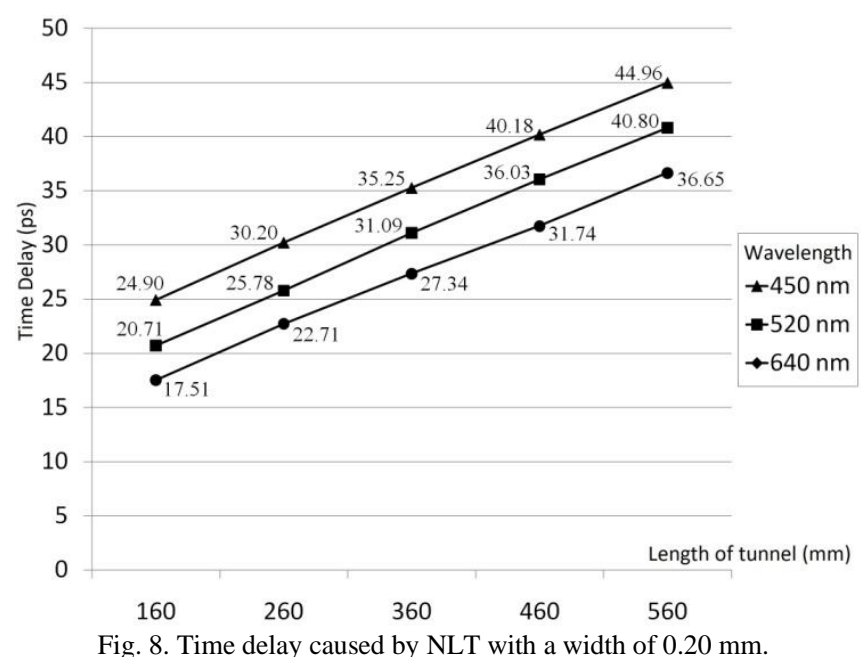

As shown by Figs. 7 and 8, the linear graphs of time delay caused by NLTs satisfy (1).

Importantly, the inaccurate dimensions of the two hundred lined up single slits with steel and plastic barriers, as well as the inaccurate dimensions of the narrow long tubes cause inaccurate values. However, the principles of the graphs as shown by Figs. 3, 7 and 8 remain the same.

\section{CONCLUSION}

This study demonstrates that the main parameters underlying the time delay causes by an aperture are the width of the gap and the thickness of the barriers (the length of hole along the optical axis).

Conversely, the experimental values show that the time delay caused by a complex of apertures and various narrow long tubes is irrespective of multiple reflections and multiple diffractions, or the possible effect is insufficient to influence the results.

In summary, considering the results presented herein, it can be inferred that the origin of the time delay caused by an aperture may lie in the fact that the refractive index of a medium is inhomogeneous and higher close to the surfaces of the obstacles, as well as the edges of the apertures. Therefore, Light propagates slowly as it travels near the surface of the object as well.

Furthermore, the inhomogeneous refractive index inside the aperture results inconsistent light speed. However, the effect of the physical dimensions of the opaque aperture on the time delay is referred to as average speed of light.

\section{ACKNOWLEDGMENT}

I am pleased to acknowledge a helpful suggestion from Dr. Jörn Schliewe for producing UDB-200/Plastic.

\section{REFERENCES}

[1] Frank Träger, Springer Handbook of Lasers and Optics, Springer New York, 2007, pp. 18.

[2] Deng-Ke Yang, Shin-Tson Wu, 2014, Fundamentals of Liquid Crystal Devices, Wiley Series in Display Technology, John Wiley \& Sons, pp. 195-196.

[3] Safa Kasap, 2006, Springer Handbook of Electronic and Photonic Materials, Springer Science \& Business Media, pp. 50-53.

[4] Vedad, F. (2021). A Geometrical Analysis of Diffraction Based on Fractal Inhomogeneous Space. European Journal of Applied Physics, $3(1), 36-42$.

[5] Joseph W. Goodman, Introduction to Fourier Optics, W. H. Freeman and Company, 2017, Chap. 2 and 3.

[6] Paul Regtien, F. van der Heijden, M. J. Korsten, W Otthius, Measurement Science for Engineers, Elsevier, 2004, pp. 283-285.

[7] Rongqing Hui, and Maurice O'Sullivan, Fiber Optic Measurement Techniques, Academic Press, 2009, pp. 32-37.

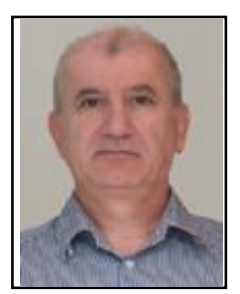

Farhad Vedad was born in Mashhad, Iran in 1963 After finishing his BSc in atomic applied physics at Tehran polytechnic university in 1994, he continued environmental engineering for a master's degree. Mr. Farhad Vedad set up a private physics lab for modern optics and meanwhile he is the author of more than twenty original articles and four books including "The Maps of Balkhi" which is trying the physics methods to solve a historical geography subject which is published by Society for the National Heritage of Iran, Experimental Investigation of Diffraction caused by Transparent Barriers. European Journal of Applied Physics, 2(5). ISSN: 2684-4451, September 19, 2020, and A Geometrical Analysis of Diffraction based on Fractal Inhomogeneous Space, European Journal of Applied Physics, 2(5). ISSN: 2684-4451, January 28, 2021. 